\title{
Statine fast für jeden, aber individualisiert
}

\author{
Der überwiegende Anteil der Männer und Frauen über 65 Jahren weist klinische Konstellatio- \\ nen auf, bei denen die Gabe eines Statins indiziert ist. Man muss sich deshalb genau überlegen, \\ welchen Personen in diesem Alter man ein Statin mit gutem Gewissen vorenthalten kann.
}

_ Knapp 30\% der US-Bevölkerung über 40 Jahren gaben 2012 an, ein Cholesterinsenkendes Medikament einzunehmen, darunter über $40 \%$ der 60 - bis 74 -Jährigen und fast die Hälfte der über 75-Jährigen. Andererseits erhalten nur $60 \%$ der Patienten mit der Aufnahmediagnose Herzinfarkt 90 Tage nach Entlassung aus dem Krankenhaus ein Statin. Wenn der Infarkt als Zweitdiagnose während des stationären Aufenthalts aufgetreten ist, waren es sogar nur 36\%, obwohl beides eine ganz klare Indikation für ein Statin darstellt.

\section{Mangelhafter Schutz nach Infarkt}

Aus aktuellen Medicare-Daten geht auch hervor, dass nur 35\% der Patienten zwischen 65 und 75 Jahren im Laufe eines Jahres nach einem akuten koronaren Ereignis ein Rezept für ein hochdosiertes Statin eingelöst haben. Nur wer schon mit hochdosierten Statinen vorbehandelt war, hatte eine hohe Chance, dass diese Therapie auch weiter fortgeführt wurde.

Die kürzlich publizierten Ergebnisse der SYNTAX-Studie zeigen eine ähnliche Situation für Deutschland. Weniger als die Hälfte der Patienten erhielten nach Bypass oder PCI eine optimale medikamentöse Therapie, nach fünf Jahren nur ein Drittel. „Irgendwie verschlafen wir alle die Sekundärprävention“, erklärte Prof. Dr. Helmut Gohlke, Ballrechten Dottingen, ehemals Herzzentrum Bad Krozingen.

\section{Sehr hohes und hohes Risiko weit verbreitet}

Die europäischen Leitlinien unterscheiden je nach dem 10-Jahres-Risiko für kar- diovaskulären Tod vier Risikokategorien, für die unterschiedliche LDL-Zielwerte festgelegt sind. Alle Patienten mit familiärer Hypercholesterinämie, dokumentierter kardiovaskulärer Erkrankung, schwerer chronischer Niereninsuffizienz und alle Diabetiker mit Risikofaktoren oder Endorganschäden gehören in die höchste Risikokategorie und brauchen ein Statin (LDL-Cholesterin-Zielwert $<70 \mathrm{mg} / \mathrm{dl}$ ). Diabetiker ohne zusätzliche Risikofaktoren, die in die Kategorie „hohes Risiko“ (Zielwert < $100 \mathrm{mg} / \mathrm{dl}$ ) gehören, gibt es eigentlich gar nicht, so Gohlke. Alle Raucher und alle Männer ab 65 Jahren - außer der systolische Blutdruck liegt unter $140 \mathrm{mmHg}$ und es gibt keine weiteren Risikomerkmale wie Übergewicht oder Bewegungsmangel gehören ebenfalls in diese Kategorie. „Natürlich gehört bei Rauchern eine Raucherentwöhnung dazu. Aber bis der Patient das geschafft hat, braucht er ein Statin“, so Gohlke. Wenn man diese Menschen alle zusammenaddiert, bleibt nur eine Minderheit, die auf Statine verzichten kann, schloss Gohlke.

\section{Individualisierte Zielwert-orientierte Therapie}

Wie Prof. Dr. Ulrich Laufs, Uniklinikum Saarland, Homburg, ergänzte, hängt der Behandlungseffekt der Statine ab vom globalen vaskulären Risiko, vom Ausgangs-LDL-Cholesterin und vom Ausmaß seiner Senkung. Wie gesund ein lebenslang niedriges LDL-Cholesterin ist, geht aus einer großen Analyse von Menschen mit genetisch niedrigem LDLCholesterin hervor. Pro $1 \mathrm{mmol}$ (ca. 40 $\mathrm{mg} / \mathrm{dl}$ ) lebenslang geringeres LDL-Cho- lesterin war das koronare Risiko um mehr als 50\% niedriger.

Das Gegenbeispiel zu diesem Idealbild am anderen Ende des Spektrums sind $\mathrm{Pa}$ tienten mit genetisch stark erhöhtem LDL-Cholesterin, d.h. familiärer Hypercholesterinämie, deren massives Risiko für kardiovaskuläre Ereignisse durch Statine deutlich gesenkt werden kann. „Es gibt wenig andere Beispiele in der Inneren Medizin, wo sich mit einer so einfachen Intervention so viel erreichen lässt", so Laufs. Diese Patienten müssen unbedingt schon als Kinder/Jugendliche behandelt werden, um die Entstehung koronarer Plaques möglichst zu verhindern.

Aber auch in der Primärprävention zahlt sich eine Statintherapie bei Menschen mit hohem LDL-Cholesterin langfristig aus. Die 16-Jahre-Follow-up-Daten der WOSCOPS-Studie ergaben für die Statingruppe im Mittel vier zusätzliche ereignisfreie Jahre. Der Endpunkt, der am stärksten beeinflusste wurde, war die Herzinsuffizienz. „Die effektivste Methode zur Prävention der Herzinsuffizienz ist, junge Leute mit einem Statin zu behandeln“, folgerte Laufs.

\section{Dr. med. Angelika Bischoff}

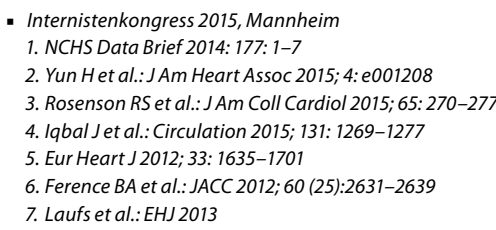

\section{FAZIT FÜR DIE PRAXIS}

Das LDL-Cholesterin sollte nicht nur so weit wie möglich, sondern auch so früh wie möglich gesenkt werden. Der größte Nutzen ist von einer Zielwert-orientierten, am individuellen Risiko ausgerichteten Therapie zu erwarten. 